\title{
Detecting mammals in heterogeneous landscapes: Implications for biodiversity monitoring and management
}

\author{
M. Swan, J. Di Stefano, F. Christie, E. Steel, A. York
}

Abstract With terrestrial mammals facing worldwide declines there is an increasing need to effectively monitor populations so that appropriate conservation actions can be taken. There are many techniques available to survey terrestrial mammals and in recent years there have been a number of studies comparing the effectiveness of different methods. Most of these studies have not considered complementarity (the degree to which techniques detect unique species) and effectiveness across ecological gradients. In this study we examined three widely used techniques, camera trapping, live trapping and hair detection, for their complementarity across a vegetation and disturbance gradient. Overall, camera trapping detected more species than any other single technique, but live trapping complemented the cameras by consistently detecting unique species. Additionally, technique effectiveness differed between vegetation types; cameras alone were most effective in dry forest systems while cameras combined with live traps were most effective in wetter forest systems. These results suggest that care needs to be taken when sampling across heterogeneous landscapes because relying on one technique alone could result in certain taxa being systematically overlooked, leading to potentially erroneous conclusions.

M. Swan*, J. Di Stefano, F. Christie, E. Steel, A. York

Department of Forest and Ecosystem Science, University of Melbourne

4 Water St Creswick, Vic 3363

*Corresponding Author

Email - $\underline{\text { mswan@student.unimelb.edu.au }}$

Keywords: Monitoring, ground-dwelling mammals, camera trapping, Elliott trapping, trapping methods, mammal detection 


\section{Introduction}

Terrestrial mammals around the world are undergoing population declines, with an estimated one fifth of all species threatened with extinction (Hoffmann et al. 2011). In many cases, efforts towards conserving species are hindered by a lack of reliable information on distributions, habitat associations and community dynamics (Rondinini et al. 2011). Such information is often difficult to obtain for terrestrial mammals because many species are rare, cryptic, and/or nocturnal (Barea-Azcon et al. 2007; Vine et al. 2009).

Accordingly, research into techniques for the effective detection of mammals is important, as the choice of technique may ultimately influence survey results and affect conservation outcomes (Garden et al. 2007). There is an increasing body of research comparing the effectiveness of different mammal survey techniques (Catling et al. 1997; Garden et al. 2007; Thompson and Thompson 2007; Vine et al. 2009; Harris and Nicol 2010; Perkins et al. 2013), including a recent focus on camera trapping relative to more traditional detection methods (Paull et al. 2012). Much of these data support the use of remote cameras as they were found to be as or more effective at detecting target mammal species when compared to live trapping (De Bondi et al. 2010), hair detection (Nelson et al. 2010; Paull et al. 2012), line transects (Silveira et al. 2003) and sign searches (Espartosa et al. 2011). Consequently, there are now many studies that have used camera trapping as the sole detection method (Ahumada et al. 2011; Rowcliffe and Carbone 2008; O'Connell et al. 2011).

Typically, studies evaluating techniques make a direct comparison of either the number of detections, detection probability, or abundance of one or more species and, sometimes, number of species detected (species richness). However, two additional factors warrant consideration. Firstly, the degree to which different techniques complement each other at a site by detecting unique species; this is especially important in the context of a biodiversity survey where the aim is to detect as many species as possible and while several studies have recommended that multiple complementary techniques are important when estimating species richness (Paull et al. 2012; Garden et al. 2007; Lyra-Jorge et al. 2008; Thompson and Thompson 2007), most have not quantified complementarity (but see Garden et al. 2007). Secondly, the degree to which external factors or covariates influence the relative effectiveness of different techniques has been overlooked. In heterogeneous landscapes in particular it is important to appreciate that the effectiveness of different techniques may not be constant and may 
be influenced by factors such as detectability of individual species, abundance, and changes in community composition along ecological gradients (Barea-Azcon et al. 2007).

In this study we use data collected with three commonly employed mammal detection methods (live trapping, hair detection and infrared camera trapping) to address two main objectives: 1 . To quantify the degree to which the three techniques complement each other by detecting unique species; and, 2 . To quantify the complementarity of the different techniques across two ecological gradients (vegetation type and time since

fire). Vegetation type and time since fire were selected a priori as they were considered to reflect biophysical changes across the study area likely to affect the composition of the mammal community (Gibson et al. 2004; Lindenmayer et al. 2008). Additionally, these two variables are highly relevant to many research and land management questions and represent ecological gradients that are frequently encountered when surveying mammals.

\section{Methods}

Study Area

The study was undertaken in a 59000 ha area of the Otway Ranges in southern Victoria, Australia (Figure 1). The area encompasses a rainfall and elevation gradient of $624 \mathrm{~mm}$ average annual rainfall near sea level in the north-east, to $1259 \mathrm{~mm}$ at $650 \mathrm{~m}$ elevation in the south-west (Bureau of Meteorology 2013). There is a corresponding change in vegetation across these gradients with predominantly treeless heath in the north-east through to wet schlerophyll forest in the south-west (Cheal 2010). Across these gradients, there is an associated change in mammal community composition (Gibson et al. 2004; Cullen 2009).

Fig. 1 Location of the Study Area, highlighting the sampling locations (mosaics) and vegetation types Study Design

Surveys took place between November 2010 and February 2011 within 32 circular, 100 ha landscapes called mosaics (Figure 1). The study area was stratified according to sections of the landscape that were dominated by five major vegetation types (Heathland, Tall-mixed Forest, Wet Forest, Foothills Forest and Forby Forest) and similar numbers of mosaics were placed in these strata using a restricted random protocol. Mosaics contained five sites, each of which consisted of a $100 \mathrm{~m}$ transect (Figure 2). Sites were located in each mosaic using a stratified random approach ensuring that all existing vegetation type - time since fire combinations were sampled. Distance between sites was set at a minimum of $100 \mathrm{~m}$ but sites were usually between 200 and $400 \mathrm{~m}$ 
apart. Six sites that were the only sampled in three minor vegetation types were removed prior to statistical analysis. The remaining 154 sites in 32 mosaics were located in six vegetation types (the five geographically dominant vegetation types and Damp Scrub) and represented a range of vegetation ages based on time since last fire (Table 1).

Table 1 Representation of vegetation types and time since fire (TSF) in the sample.

\begin{tabular}{lccc}
\hline Vegetation Type & $\begin{array}{c}\text { Number of } \\
\text { Mosaics }\end{array}$ & $\begin{array}{c}\text { Number } \\
\text { of Sites }\end{array}$ & $\begin{array}{c}\text { TSF Range } \\
\text { (Years) }\end{array}$ \\
\hline Heathland & 9 & 24 & $0-28$ \\
Tall Mixed Forest & 12 & 28 & $0-45$ \\
Foothills Forest & 13 & 33 & $0-72$ \\
Forby Forest & 6 & 23 & $0-72$ \\
Wet Forest & 16 & 38 & $0-72$ \\
Damp Scrub & 6 & 8 & $0-28$ \\
\hline
\end{tabular}

\section{Live Trapping}

Ten small (Type A: 9 × $10 \times 33 \mathrm{~cm}$ ) and two large (Type E: $15 \times 15.5$ x $46 \mathrm{~cm}$ ) Elliott traps were used to target small and medium sized terrestrial mammals. Traps were placed along each transect at approximately eight metre intervals with the two large traps at the $20 \mathrm{~m}$ and $80 \mathrm{~m}$ marks (Figure 2). In accordance with the recommendations of Tasker and Dickman (2001) traps were placed in locations considered likely to be encountered by animals such as adjacent to logs, in runways or in dense vegetation with the proviso that they were within 5 metres of the transect. Traps were baited with rolled oats, peanut butter, golden syrup and pistachio essence, which has been shown to be effective for detecting a wide range of ground-dwelling mammals (Paull et al. 2011). A handful of unbleached cotton was added to each trap to provide insulation. In wet weather traps were covered with plastic bags for protection. Trapping took place for three consecutive nights at each site. Animals caught were identified to species level, sexed, temporarily marked with liquid paper on the base of the tail, and released at the point of capture.

\section{Hair surveys}

We used tapered hair funnels (Faunatech Pty Ltd, Bairnsdale, Victoria) to target small, medium and large mammals. Five hair funnels were placed at 20 metre intervals starting at the $20 \mathrm{~m}$ mark of each transect and left in place for between 19 and 22 days. Hair funnels were baited with the same bait as for live trapping. At the end of the survey period hair samples were sent to an expert (Barbara Triggs) for identification.

\section{Camera trapping}


One Scoutguard 550v and one Reconyx HC500 were deployed at each site at the $20 \mathrm{~m}$ and $80 \mathrm{~m}$ mark of the $100 \mathrm{~m}$ transect, respectively. Both of these models have been used extensively in fauna surveys (De Bondi et al. 2010; Robley et al. 2011; Kelly and Holub 2008) and deploying two cameras per site has been shown to be a robust design (Negroes et al. 2012). We followed the camera setup protocols outlined in Nelson and Scroggie (2009) for surveying mammals of different sizes. Each camera was mounted approximately $50 \mathrm{~cm}$ above the ground on a tree and a bait station was placed approximately $2.5 \mathrm{~m}$ in front of each camera. The bait station consisted of five tea infusers containing bait (as above) suspended approximately $30 \mathrm{~cm}$ off the ground on a wooden stake. Cameras were oriented in a southerly direction to minimise the influence of afternoon sun on trigger events. Vegetation between the camera and bait station was cleared to improve identification capacity. Both camera types were set to their highest sensitivity rating (very high on a five point scale for Reconyx and high on a three point scale for Scoutguard), and to take three photographs on sensor activation with a one minute delay between events. Cameras were left in place for 19 to 26 days. For subsequent analysis, all records of species detected after 19 days were disregarded.

Fauna captured in photos were identified to species by four researchers with similar levels of experience. We used a reference guide (Menkhorst and Knight 2004) and a library of accumulated images to aid species' identification. For each photo event (defined as the group of three photos resulting from each sensor activation) we recorded the species, date, time of day, and number of individuals. Individuals of Rattus spp. and Antechinus spp. could only consistently be identified to genus. Consequently, we grouped small mammal records by genera for each survey method.

Hair and camera surveys were conducted concurrently. Live trapping was conducted immediately before or after the passive surveys to remove the potential for activities associated with trapping to reduce the effectiveness of the other two techniques. The small numbers of arboreal mammal species detected during surveys were removed from the data set prior to analysis.

Fig. 2 Layout of the three different sampling techniques at each site within each mosaic Statistical analysis

We calculated species richness (number of species detected) at the site level for each of the three techniques independently, and also for all possible combinations of different techniques $(n=7)$. Including the combinations of the multiple techniques allowed us to assess the degree to which the different techniques 
complemented each other by detecting additional species. Statistical modelling took place in two stages. In stage one, we used a Generalised Linear Mixed Model (GLMM) assuming a poisson error structure to quantify the overall effect of technique (including the three individual techniques and their combinations) on species richness. This was undertaken at the site scale with mosaic included as a random term in the model to reflect the spatially nested study design.

In stage two we tested the relative effectiveness of the different techniques along ecological gradients by modelling interactions between technique and two landscape variables, vegetation type and time since last fire. We used the same modelling framework as before but compared three alternative models: 1. technique, 2. technique $\times$ vegetation type, 3 . technique $\times$ time since last fire. We did not run models of technique in additive combinations with time since last fire and vegetation type as these reflected patterns of change in species richness that were not of primary interest. Values for time since fire were not absolute values (i.e. years) but an index ranging from $0-1$ to account for the differing developmental rates exhibited by the different vegetation types (Cheal 2010). For example, in this system Heathland is considered mature by 30 years of age, whereas 30 year old wet forest is still considered adolescent (Cheal 2010). Models were compared using an information theoretic approach where those with lower values of Akaike's Information Criterion (AIC) were considered more parsimonious (Burnham and Anderson 2002). Akaike weights were calculated to indicate the relative likelihood that a particular model was the best in the set (Burnham and Anderson 2002). We calculated variance explained $\left(\mathrm{R}^{2}\right)$ as a measure of model fit using the methods outlined in Nakagawa and Schielzeth (2013) where marginal $\mathrm{R}^{\mathbf{2}}$ values refer to the variance explained by the fixed factors alone and conditional $\mathrm{R}^{\mathbf{2}}$ is the variance explained by both the fixed and random effects. We used the R Statistical Program version 2.15.3 (R Core Team 2013) and GenStat version 14 (VSN International 2011) for analysis.

\section{Results}

We recorded 19 ground-dwelling mammal species with the three different techniques. Camera trapping recorded 13 species, live trapping 5 species and hair detection 11 species. Each technique detected at least one species that the other techniques failed to detect (Table 1). Overall, the camera trapping recorded more species per site than live traps $(\mathrm{p}<0.01)$ and hair detection $(\mathrm{p}<0.01)$ and live trapping recorded more species than the hair detection $(\mathrm{p}<0.01)$ (Figure 3). 
Complementarity

When all three techniques were combined they detected 2.9 species on average per site (Figure 3 ), which was $28 \%$ (0.65 species, $95 \%$ CI $0.53-0.76, \mathrm{p}<0.01)$ greater than cameras alone. However, there was no difference between the number of species detected by the three techniques combined and cameras + Elliott traps $(0.06$, $95 \%$ CI $-0.05-0.17, \mathrm{p}=0.284)$. There was a small but statistically significant difference between Elliott traps + hair funnels and Elliott traps alone $(0.24,95 \%$ CI $0.06-0.42, \mathrm{p}<0.01)$.

Table 2 Number of sites out of a total of 154 where species were positively identified with each technique including detections of different genera of small mammals.

\begin{tabular}{llccc}
\hline Species name & Common Name & Camera & Elliott & Hair \\
\hline Macropus giganteus & Eastern Grey Kangaroo & 33 & 0 & 0 \\
Macropus rufogriseus & Red-necked Wallaby & 4 & 0 & 0 \\
Wallabia bicolor & Swamp Wallaby & 132 & 0 & 15 \\
Potorous tridactylus & Long-nosed Potoroo & 10 & 0 & 1 \\
Tachyglossus aculeatus & Short-beaked Echidna & 60 & 0 & 4 \\
Isodon obesulus & Southern-brown Bandicoot & 6 & 0 & 0 \\
Perameles nasuta & Long-nosed Bandicoot & 7 & 0 & 0 \\
Rattus fuscipes & Bush Rat & 20 & 72 & 21 \\
Rattus lutreolus & Swamp Rat & 1 & 8 & 5 \\
Rattus rattus & Black Rat & 0 & 3 & 3 \\
Antechinus agilis & Agile Antechinus & 8 & 68 & 16 \\
Antechinus swainsonii & Dusky Antechinus & 5 & 26 & 1 \\
Antechinus minimus & Swamp Antechinus & 0 & 0 & 4 \\
Mus domesticus* & House Mouse & 0 & 0 & 4 \\
Felis catus & Cat & 14 & 0 & 1 \\
Vulpes vulpes & Red Fox & 25 & 0 & 0 \\
Genus level for small & & & & \\
mammals & & & & \\
\hline Rattus spp. & & 48 & 75 & 29 \\
Antechinus spp. & & 28 & 75 & 21 \\
\hline *exotic species & & & &
\end{tabular}

*exotic species

Fig. 3 Generalised linear mixed model predictions of species richness with three independent techniques and four combinations of techniques. $\mathrm{C}=$ Camera Trap, $\mathrm{E}=$ Elliott Trap and $\mathrm{H}=$ Hair Funnel. Error bars are $95 \%$ Confidence intervals

The Effect of Vegetation Type and Time Since Last Fire

The model including the interaction between technique and vegetation type was clearly the best of the three considered (Table 2; Akaike weight $=0.99$, Marginal $\mathrm{R}^{2}=0.56$ ) suggesting that the relative performance of the techniques differed between vegetation types. 
Table 3 Model selection statistics for species richness. Models were ranked using Akaike's information criterion (AIC).

\begin{tabular}{lccccc}
\hline Models & AIC & AAIC & $\begin{array}{c}\text { Akaike } \\
\text { weight }(\mathbf{w})\end{array}$ & $\begin{array}{c}\text { Marginal } \\
\mathbf{R}^{\mathbf{2}}\end{array}$ & $\begin{array}{c}\text { Conditional } \\
\mathbf{R}^{\mathbf{2}}\end{array}$ \\
\hline Technique * Vegetation Type & 789.4 & 0 & 0.99 & 0.56 & 0.64 \\
Technique & 827.4 & 38 & 0.00 & 0.42 & 0.55 \\
Technique * Time Since Fire & 834.8 & 45.4 & 0.00 & 0.42 & 0.55 \\
\hline
\end{tabular}

In Damp Scrub, Heathland and Tall Mixed Forest there was very little difference between cameras alone and cameras + Elliott traps + hair funnels (Figure 4). In Wet Forest, Foothills Forest and Forby forest there were significant differences of 0.77 (95\% CI $0.55-0.97, \mathrm{p}<0.01), 1.07$ (95\% CI $0.82-1.3, \mathrm{p}<0.01)$ and $0.75(95 \%$ CI $0.48-1.02, \mathrm{p}<0.01)$ species respectively between cameras alone and cameras + Elliott traps + hair funnels

(Figure 4). In these three vegetation types there was negligible difference between cameras + Elliott traps + hair funnels and cameras + Elliott traps. The model including the interaction between time since fire and technique was the worst in the set with a very small Akaike weight.

Fig. 4 Generalised linear mixed model predictions of species richness for each combination of techniques in the six different vegetation types. a) Damp Scrub, b) Forby Forest, c) Tall Mixed Forest d) Foothills Forest e) Heathland f) Wet Forest. $\mathrm{C}=$ Camera Trap, $\mathrm{E}=$ Elliott Trap and $\mathrm{H}=$ Hair Funnel. Error bars are $95 \%$ Confidence intervals

\section{Discussion}

In recent years there has been an increasing interest in the effectiveness of different sampling methods for detecting mammal species (Paull et al. 2012). This study is the first to investigate the effectiveness of live trapping, hair detection and camera trapping concurrently. The findings of this study are consistent with previous studies that have found camera traps to be an effective technique for detecting a range of species of different body sizes (De Bondi et al. 2010; Espartosa et al. 2011; Paull et al. 2012; Silveira et al. 2003). The other two techniques were found to be less effective than the cameras in terms of the total number of species detected per site. This result was expected for the Elliott traps as they were only capable of capturing small to medium mammals, whereas the cameras and hair funnels could capture mammals of any size, thus sampling from a larger pool of species. Of greater interest is the ability of the techniques to complement each other by detecting unique species (Garden et al. 2007).

Complementarity of Techniques 
All three techniques detected at least one unique species that the other techniques failed to detect; therefore each technique complemented at least one other technique to some degree. At the site level the cameras detected the most species, but live trapping complemented them by adding on average 0.6 species per site over and above the cameras alone. This result was largely driven by the small mammal fauna of the study area. The Elliott traps were consistently more effective at detecting Antechinus spp. (detected at 75 sites with Elliott's vs. 28 sites with cameras) and to a lesser extent Rattus spp. (detected at 75 sites with Elliott's vs. 48 sites with cameras), despite being deployed for a shorter length of time than the cameras. Although the larger Type E Elliott traps were capable of trapping medium-sized mammals (e.g. potoroos and bandicoots), none of these species were trapped, therefore Type E traps were effectively equivalent to the small Type A traps. Medium-sized species have been found to be trap shy with infrared cameras proving a more effective detection method than live trapping (Claridge et al. 2010). However, it is possible that a longer trap deployment period may have increased captures of medium sized species.

In considering our small mammal detections, the disparity between cameras and live trapping is inconsistent with recent research that has shown camera traps to be as effective as live trapping for detecting small mammals (De Bondi et al. 2010). There are a number of potential explanations for this. First, we found the Scoutguard brand Cameras to be very poor at detecting small mammals compared to the Reconyx, therefore we effectively had one small mammal sampling camera instead of two. The two cameras types consistently detected the same suite of large mammal species at a single site, however it is unknown if two Reconyx would have performed better than one in terms of small mammal detections. Second, the alteration of microhabitat as part of the camera set up protocol may have negatively affected species behaviour thus reducing detectability. The Elliott traps were placed in microhabitat that was deemed likely to render captures such as next to logs or within dense vegetation. Comparatively, setting up cameras involved the removal of aboveground biomass and often significant alteration of habitat. Whilst the removal of vegetation enhanced our capacity to obtain clear images of animals for identification, it is possible that small mammals actively avoided such open areas (Garden et al. 2007). Third, by targeting mammals of all sizes we may have set the bait station too far away from the camera for small species heat signals to be consistently detected by the camera. High detection rates of small mammals have been observed with cameras located closer to the bait station (Paull et al. 2012). A trade-off may therefore exist between detecting species of all body sizes and detecting small mammals specifically. Setting the camera closer to the bait station could facilitate a greater number of small mammal detections but it is not known if this 
would affect the ability to identify large species due to a reduced field of view. Our cameras were oriented horizontally, however, downward facing cameras have recently shown promise in the ability to detect and identify small mammals (De Bondi et al. 2010; Smith and Coulson 2012). One drawback of a downwards orientation, however, is that it reduces the size of the camera's detection zone and so has the potential to miss individuals that do not approach the bait station directly. Further research is warranted into determining the most effective camera trap setups for detecting multiple species of different body sizes and exhibiting different behaviours.

Unlike live trapping, the hair funnels did not complement the camera trapping by detecting unique species consistently. There was some evidence that they provided limited complementary information to Elliot traps, but this is attributed to the hair funnels detecting larger bodied species. The superiority of camera traps over hair detection devices is consistent with a recent study that compared these techniques (Paull et al. 2012). With the increasing evidence of the effectiveness of camera trapping, the utility of hair detection devices will likely be superseded (Paull et al. 2012). This is further compounded by other advantages of camera traps such as the ability to calculate detection probability, the ability to garner behavioural information, and the estimation of abundance in some circumstances (Nelson et al. 2010; Paull et al. 2012; Rowcliffe et al. 2008). The only value of the hair funnels in our study was the detection of two species that the other techniques failed to detect. One of these species, the Swamp Antechinus Antechinus minimus, is a listed threatened species that was recorded at four sites. It is possible that hair detection devices may be of use in detecting such threatened species that are not easily trapped and occur at low densities, thus requiring a large number of sampling units to detect them. The relatively low cost of hair funnels may make them a viable alternative to more costly camera trapping when high sampling intensity is required.

The Effect of Vegetation Type

To our knowledge, we are the first to systematically compare different mammal sampling techniques in different vegetation types and successional stages. Vegetation type is known to be a strong driver of mammal community composition (Williams et al. 2002) and our study has shown that this can influence the relative effectiveness of different sampling techniques. In the drier vegetation types, Heathland, Tall Mixed Forest and Damp Scrub the use of cameras alone would have been sufficient, with other techniques providing mostly redundant information. In the wetter, more productive Foothills Forest, Wet Forest and Forby Forest the combination of Elliott traps 
and camera traps was most effective, with the former complementing the latter by adding approximately one species per site. These results were largely driven by the distribution of small mammals across the landscape. In the drier forest systems there were fewer small mammals and thus cameras detected almost all species present. In wetter vegetation types small mammals were much more widespread and abundant, resulting in substantial complementary information from live trapping. The absence of live trapping in the wetter forest systems would have resulted in a systematic underestimation of species richness in these areas.

\section{Cost-benefit considerations}

Decisions about which techniques to deploy are often limited by practical considerations, most notably cost (De Bondi et al. 2010; Garden et al. 2007; Paull et al. 2012). Accordingly, much research has focussed on cost benefit analyses between different techniques. In this study we have not attempted to do this because costs are project specific and depend on variables such as the distance between sites, the use of student versus staff labour and costs of transport, which vary markedly between institutions. Nevertheless, indirect techniques such as camera trapping may often be cheaper than live trapping due to lower labour and travel costs, and they therefore have the potential to be deployed for greater periods of time and at a greater number of sites (De Bondi et al. 2010). This may provide further incentive for practitioners to use cameras alone and ignore more expensive techniques. However one factor that is not often considered in this type of analysis is the potential costs of not recording species. In the present study we have shown that although cameras were effective at detecting multiple species, using them alone would have lead to us to underestimate species richness in certain parts of the landscape. Erroneous conclusions such as this could lead to inappropriate management actions and allocation of resources, the costs of which are potentially large but difficult to quantify.

\section{Conclusion}

In this study we found that live trapping complemented camera trapping by consistently detecting unique species, especially in wetter forest types where small mammals were more widespread and abundant. We argue that caution needs to be taken in only using one technique alone due to the potential to miss certain species or groups of species in certain habitat types. Decisions about the techniques to employ are made especially difficult when surveys are implemented in large, heterogeneous landscapes where it is not always possible to predict a priori what species will occur and which techniques will be most suitable. The most effective technique or combination of techniques will depend on the characteristics of the study area and the biological 
assemblage that it supports. A precautionary approach using appropriate pilot studies and adaptive monitoring principles could be useful in this context (Lindenmayer and Likens 2009). This would involve constantly evaluating the performance of different techniques for the duration of monitoring programs and adapting as necessary. This should be complemented by further research into the effectiveness of techniques across different habitat types. 


\section{Acknowledgments}

We thank Michelle Ibbett, Holly Sitters, Evan Watkins and Leigh Morison for assistance in the field and Amanda Ashton and Helen Doherty for help with photo processing. This research was supported by the Department of Environment and Primary Industries (DEPI), Parks Victoria and the Holsworth Wildlife Research Endowment. Field work was conducted under the National Parks Act (Research Permit Number 10005348) and Forests Act (Scientific Permit Number 10005514), and faunal surveys were approved by the University of Melbourne School of Land and Environment Ethics Committee (Register No. 1011632.5). 


\section{References}

Ahumada JA, Silva CEF, Gajapersad K, Hallam C, Hurtado J, Martin E, McWilliam A, Mugerwa B, O'Brien T, Rovero F, Sheil D, Spironello WR, Winarni N, Andelman SJ (2011) Community structure and diversity of tropical forest mammals: data from a global camera trap network. Philos Trans R Soc B: Biol Sci 366 (1578):2703-2711. doi:10.1098/rstb.2011.0115

Barea-Azcon JM, Virgos E, Ballesteros-Duperon E, Moleon M, Chirosa M (2007) Surveying carnivores at large spatial scales: a comparison of four broad-applied methods. Biodivers Conserv 16 (4):1213-1230.

doi:10.1007/s10531-006-9114-x

Bureau of Meteorology (2013) Climate statistics for Australian Locations. http://www.bom.gov.au/climate/data/?ref=ftr. Accessed March 2013

Burnham KP, Anderson DR (2002) Model selection and multimodel inference: a practical information-theoretic approach. Springer, New York

Catling PC, Burt RJ, Kooyman R (1997) A Comparison of Techniques Used in a Survey of the Grounddwelling and Arboreal Mammals in Forests in North-eastern New South Wales. Wildlife Res 24 (4):417-432. doi:http://dx.doi.org/10.1071/WR96073

Cheal D (2010) Growth stages and tolerable fire intervals for Victoria's native vegetation datasets. Fire and Adaptive Management Report No. 84. Department of Sustainability and Environment, East Melbourne, Victoria

Claridge AW, Paull DJ, Barry SC (2010) Detection of medium-sized ground-dwelling mammals using infrared digital cameras: an alternative way forward? Aust Mammal 32 (2):165-171 doi:10.1071/am09039

Cullen M (2009) Vascular plant and small mammal communities over an elevational gradient. PhD Thesis, Deakin University,

De Bondi N, White JG, Stevens M, Cooke R (2010) A comparison of the effectiveness of camera trapping and live trapping for sampling terrestrial small-mammal communities. Wildlife Res 37 (6):456-465. doi:10.1071/wr10046

Espartosa KD, Pinotti BT, Pardini R (2011) Performance of camera trapping and track counts for surveying large mammals in rainforest remnants. Biodivers Conserv 20 (12):2815-2829. doi:10.1007/s10531-011-0110-4

Garden JG, McAlpine CA, Possingham HP, Jones DN (2007) Using multiple survey methods to detect terrestrial reptiles and mammals: what are the most successful and cost-efficient combinations? Wildlife Res 34 (3):218-227. doi:10.1071/wr06111

Gibson LA, Wilson BA, Aberton JG (2004) Landscape characteristics associated with species richness and occurence of small native mammals inhabiting coastal heathland: a spatial modelling approach. Biol Conserv 120:75-89

Harris RL, Nicol SC (2010) The effectiveness of hair traps for surveying mammals: results of a study in sandstone caves in the Tasmanian southern midlands. Aust Mammal 32 (1):62-66. doi:doi:10.1071/AM09019

Hoffmann M, Belant JL, Chanson JS, Cox NA, Lamoreux J, Rodrigues ASL, Schipper J, Stuart SN (2011) The changing fates of the world's mammals. Philosophical Transactions of the Royal Society B-Biological Sciences 366 (1578):2598-2610. doi:10.1098/rstb.2011.0116

Kelly MJ, Holub EL (2008) Camera Trapping of Carnivores: Trap Success among Camera Types and across Species, and Habitat Selection by Species, on Salt Pond Mountain, Giles County, Virginia. Northeast Nat 15 (2):249-262 
Lindenmayer DB, Likens GE (2009) Adaptive monitoring: a new paradigm for long-term research and monitoring. Trends Ecol Evol 24 (9):482-486. doi:10.1016/j.tree.2009.03.005

Lindenmayer DB, MacGregor C, Welsh A, Donnelly C, Crane M, Michael D, Montague-Drake R, Cunningham RB, Brown D, Fortescue M, Dexter N, Hudson M, Gill AM (2008) Contrasting mammal responses to vegetation type and fire. Wildlife Res 35 (5):395-408. doi:10.1071/wr07156

Lyra-Jorge MC, Ciocheti G, Pivello VR, Meirelles ST (2008) Comparing methods for sampling large- and medium-sized mammals: camera traps and track plots. Eur J Wildl Res 54 (4):739-744. doi:10.1007/s10344008-0205-8

Menkhorst P, Knight F (2004) A Field Guide to the Mammals of Australia. Oxford University Press, Melbourne

Nakagawa S, Schielzeth H (2013) A general and simple method for obtaining R2 from generalized linear mixedeffects models. Methods Ecol Evol 4 (2):133-142. doi:10.1111/j.2041-210x.2012.00261.x

Negroes N, Sollmann R, Fonseca C, Jacomo ATA, Revilla E, Silveira L (2012) One or two cameras per station? Monitoring jaguars and other mammals in the Amazon. Ecol Res 27 (3):639-648. doi:10.1007/s11284-0120938-4

Nelson J, Main M, Chick R, Scroggie M (2010) The Status of Smoky Mouse populations at historic sites in Victoria, and an assessment of two non-invasive survey techniques. Unpublished report to the Department of Environment, Water, Heritage and the Arts, and the Goulburn Broken Catchment Management Authority. Department of Sustainability and Environment, Heidelberg, Victoria

Nelson JL, Scroggie MP (2009) Remote cameras as a mammal survey tool - survey design and practical considerations. . Arthur Rylah Institute for Environmental Research Unpublished Report No. 2009/36. Department of Sustainability and Environment, Heidelberg, Victoria

O'Connell AF, Nichols JD, Karanth KU (2011) Camera Traps in Animal Ecology. Springer, Tokyo

Paull DJ, Claridge AW, Barry SC (2011) There's no accounting for taste: bait attractants and infrared digital cameras for detecting small to medium ground-dwelling mammals. Wildlife Res 38 (3):188-195. doi:10.1071/wr10203

Paull DJ, Claridge AW, Cunningham RB (2012) Effective detection methods for medium-sized grounddwelling mammals: a comparison between infrared digital cameras and hair tunnels. Wildlife Res 39 (6):546553. doi:http://dx.doi.org/10.1071/WR12034

Perkins GC, Kutt AS, Vanderduys EP, Perry JJ (2013) Evaluating the costs and sampling adequacy of a vertebrate monitoring program. Australian Zoologist 36 (3):373-380

R Core Team (2013) R: A language and environment for statistical computing. R Foundation for Statistical Computing, Vienna, Austria. http://www.R-project.org/

Robley A, Gormley A, Albert R, Bowd M, Hatfield C, McDonald R, Thorp A, Scroggie M, Smith A, Warton F (2011) Glenelg Ark 2005-2010: evidence of sustained control of foxes and benefits for native mammals. Arthur Rylah Institute for Environmental Research Technical Report Series No. 226. Department of Sustainability and Environment, Heidelberg, Victoria

Rondinini C, Rodrigues ASL, Boitani L (2011) The key elements of a comprehensive global mammal conservation strategy. Philos Trans R Soc B: Biol Sci 366 (1578):2591-2597. doi:10.1098/rstb.2011.0111

Rowcliffe JM, Carbone C (2008) Surveys using camera traps: are we looking to a brighter future? Anim Conserv 11 (3):185-186. doi:10.1111/j.1469-1795.2008.00180.x

Rowcliffe JM, Field J, Turvey ST, Carbone C (2008) Estimating animal density using camera traps without the need for individual recognition. J Appl Ecol 45 (4):1228-1236. doi:10.1111/j.1365-2664.2008.01473.x 
Silveira L, Jácomo ATA, Diniz-Filho JAF (2003) Camera trap, line transect census and track surveys: a comparative evaluation. Biol Conserv 114 (3):351-355

Smith JK, Coulson G (2012) A comparison of vertical and horizontal camera trap orientations for detection of potoroos and bandicoots. Aust Mammal 34 (2):196-201. doi:10.1071/am11034

Tasker E, Dickman C (2001) A review of Elliott trapping methods for small mammals in Australia. Aust Mammal 23 (2):77-87. doi:http://dx.doi.org/10.1071/AM01077

Thompson GG, Thompson SA (2007) Usefulness of funnel traps in catching small reptiles and mammals, with comments on the effectiveness of the alternatives. Wildlife Res 34 (6):491-497. doi:10.1071/wr06081

Vine SJ, Crowther MS, Lapidge SJ, Dickman CR, Mooney N, Piggott MP, English AW (2009) Comparison of methods to detect rare and cryptic species: a case study using the red fox (Vulpes vulpes). Wildlife Res 36 (5):436-446. doi:http://dx.doi.org/10.1071/WR08069

VSN International (2011) GenStat for Windows 14th Edition. Hemel Hampstead, UK. Genstat.co.uk

Williams SE, Marsh H, Winter J (2002) Spatial scale, species diversity, and habitat structure: small mammals in australian tropical rain forest. Ecology 83 (5):1317-1329. doi:10.2307/3071946

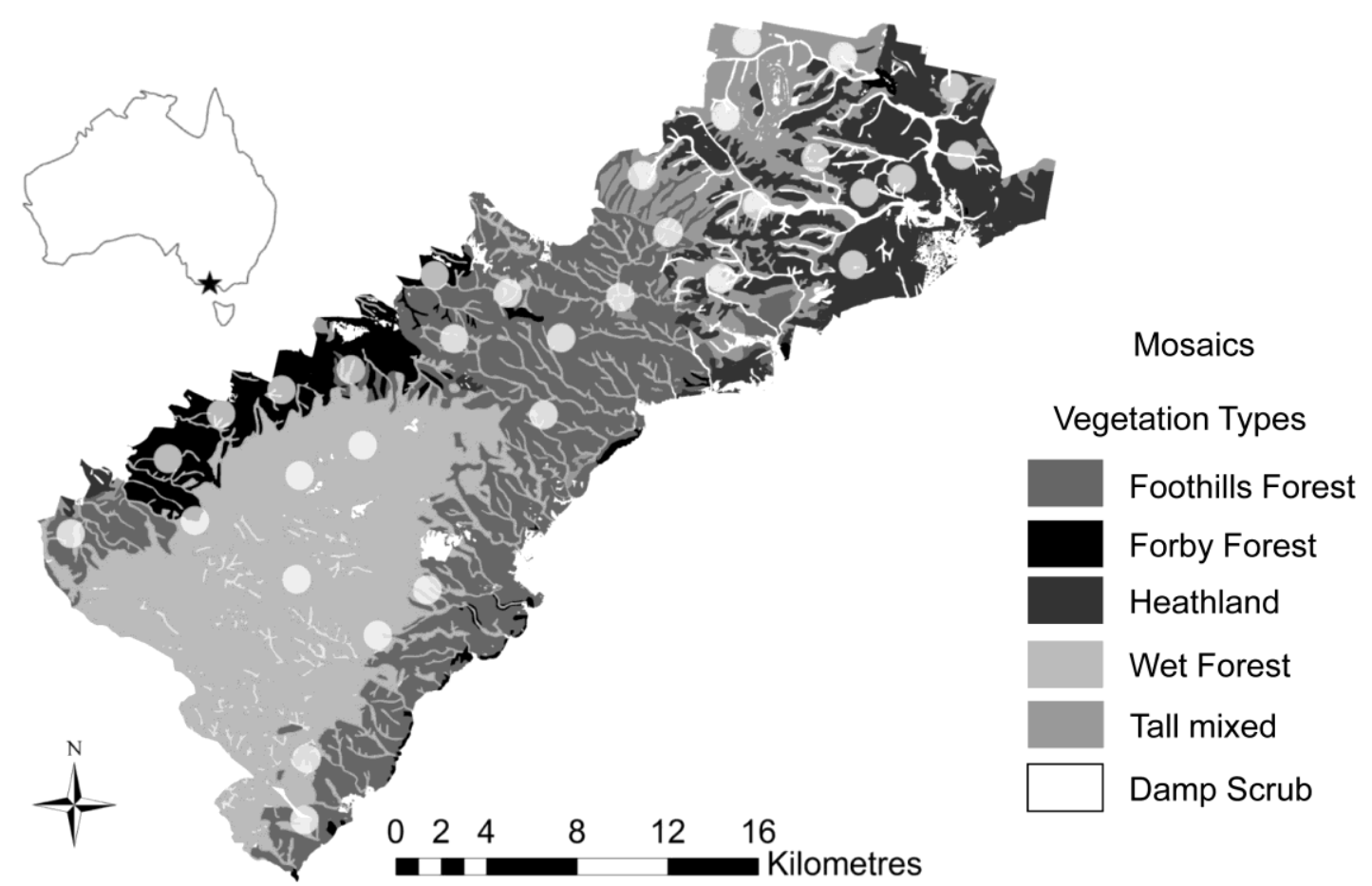




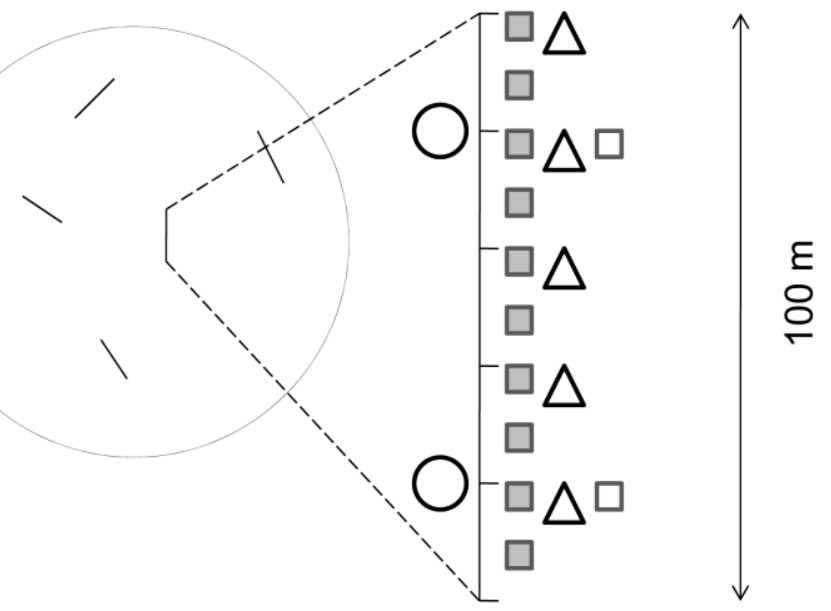
$\triangle$ Hair Funnel
Camera Trap
$\square$ Small Elliott Trap
$\square$ Large Elliott Trap

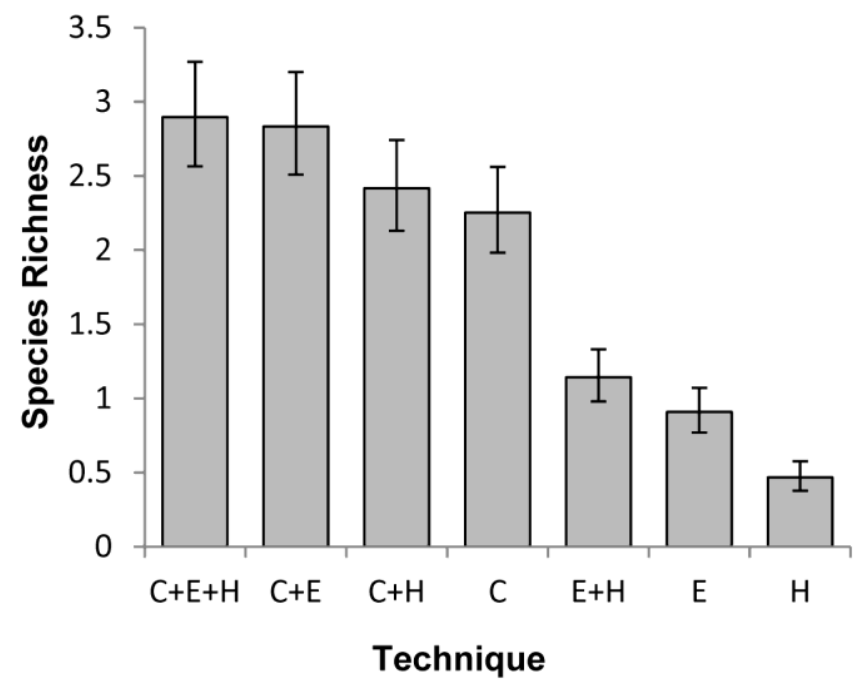



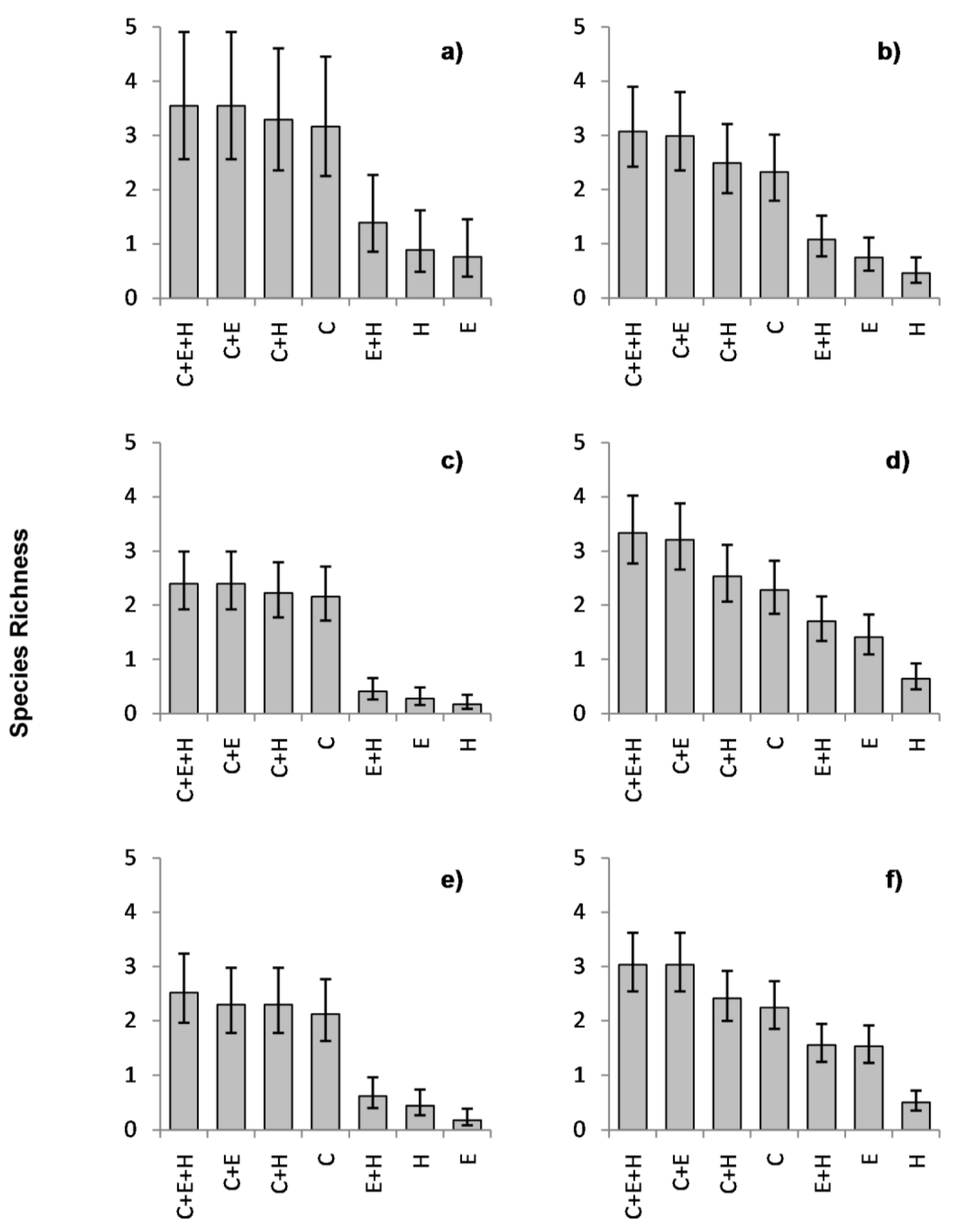

Technique 


\section{University Library}

\section{- M M N E R VA A gateway to Melbourne's research publications}

Minerva Access is the Institutional Repository of The University of Melbourne

Author/s:

Swan, M;Di Stefano, J;Christie, F;Steel, E;York, A

Title:

Detecting mammals in heterogeneous landscapes: implications for biodiversity monitoring and management

Date:

2014-02-01

Citation:

Swan, M., Di Stefano, J., Christie, F., Steel, E. \& York, A. (2014). Detecting mammals in heterogeneous landscapes: implications for biodiversity monitoring and management. BIODIVERSITY AND CONSERVATION, 23 (2), pp.343-355. https://doi.org/10.1007/ s10531-013-0604-3.

Persistent Link:

http://hdl.handle.net/11343/283160 